UDC $616.72-002-053.2 / 5:$ 616-056.2

DOI: $10.15587 / 2519-4798.2020 .213126$

\title{
FIBROBLAST GROWTH FACTOR AND HEPATOCYTE GROWTH FACTOR IN ADOLESCENTS WITH JUVENILE IDIOPATHIC ARTHRITIS TREATED WITH METHOTREXATE
}

\section{O. Pavlova}

Methotrexate (MTX) is a cornerstone of therapy worldwide for juvenile idiopathic arthritis (JIA). Despite the fact that fibrosis molecular mechanisms as well as MTX elimination and fibrosis indexes were studied a lot there is still not enough information for adolescence.

The aim was to study dynamics of molecular-cellular mechanisms activation of fibrotic processes development in the liver in adolescents with juvenile idiopathic arthritis treated with methotrexate by determining the content of fibroblast growth factor and hepatocyte growth factor.

Materials and methods: A total of 68 children with juvenile idiopathic arthritis, were enrolled in the study. 25 boys (36.8\%) and 43 girls (63.2\%) were examined. Children were divided into four groups in accordance with cumulative dose (CD) of methotrexate. The following data were analyzed: liver function tests (aspartate aminotransferase (AST) (U/L), alanylaminotransferase (ALT) (U/L)), lactate dehydrogenase (LDH) (U/L), adiponectin $(\mu \mathrm{g} / \mathrm{ml}), B F G F(\mathrm{pg} / \mathrm{ml}), H G F(\mathrm{pg} / \mathrm{ml})$, liver fibrosis indexes APRI and FIB-4 Score.

Results. Positive effect of JIA treatment with MTX on the liver is noted. When CD MTX reaches 1 and 3 grams, liver state studying is needed. When the CD MTX of 1 gram is reached, regulatory mechanisms are involved that provoke liver regeneration. When the CD MTX reaches 3 grams, the liver condition may deteriorate, which in the future can lead to irreversible processes of liver fibrosis.

Conclusions: Thus, it is important to control possible liver disorders in adolescence treated with MTX. Monitoring the processes of liver fibrosis is appropriate at all stages of JIA treatment, but it is most advisable when the MTX cumulative dose is reaching 1 and 3 grams

Keywords: juvenile idiopathic arthritis, methotrexate, fibroblast growth factor, hepatocyte growth factor, adiponectin, APRI index, FIB-4 Score, liver ultrasound, adolescents, liver

Copyright (C) 2020, O. Pavlova.

This is an open access article under the CC BY license (http://creativecommons.org/licenses/by/4.0).

\section{Introduction}

Juvenile idiopathic arthritis (JIA) is the most common rheumatic disease of childhood (1:1000 children). Methotrexate (MTX) remains the drug of choice for JIA treatment, despite the development and active implementation of immunobiological therapy [1]. Methotrexate is commonly administered orally as a single weekly dose during JIA treatment. MTX dosage is clearly regulated by modern treatment guidelines. After oral administration, MTX is absorbed in the proximal jejunum by the proton-coupled folate transporter (PCFT/SLC46A1), which transports reduced folates and MTX [2]. Major elimination route of MTX is renal excretion constitutes. Firstpass of MTX metabolism occurs in the liver, where it is converted to 7-hydroxymethotrexate (7-OH-MTX), which is major metabolite of MTX [3].

Hepatotoxicity remains one of the most common side effects of MTX. Slight increase in aminotransferases level, hepatic steatosis, fibrosis and cirrhosis are most common manifestations of hepatotoxicity [4], which reflect the morphological features of non-alcoholic fatty liver disease (NAFLD). NAFLD pathogenesis associated with MTX treatment mostly occurs in such ways:

1) by MTX ability to enhance adenosine release from cultured cells, which further stimulates collagen production by stellate cells of the liver $[5,6]$.
2) MTX also interferes with the generation of methionine from homocysteine. Excess homocysteine promotes fat accumulation in the liver and can activate proinflammatory cytokines and hepatic stellate cells, leading to liver fibrosis $[7,8]$.

3) may be associated with reactive oxygen species generation. MTX has been shown to cause oxidative tissue damage by enhancing lipid peroxidation in liver tissues and reducing the activity of antioxidant enzymes [9].

Due to the significant compensatory potential of the liver in childhood, it is important to prevent the development of steatosis or fibrosis in the future in these patients. Including the study of the risk of fibrosis is possible using trigger factors for its development.

Fibroblast growth factor (FGF) has shown a potential effect on tissue repair and regeneration [10, 11]. It was originally identified as a protein capable of stimulating fibroblast proliferation. It is now known that the group of these factors includes 22 members, of which FGFs perform multiple functions by binding and activating fibroblast growth factor receptors (FGFR). Fibroblast growth factor receptors 1, 2 and 4 are expressed in the liver, and FGFR4 exclusively in hepatocytes [12]. FGF1 and FGF2 are produced during hepatocyte replication and are mitogens for both endothelial and stellate liver cells [13]. 
Hepatocyte growth factor (HGF) was first identified as a mitogenic protein for rat hepatocytes in 1984 [14]. The proliferative activity of hepatocytes is associated with the level of organ-specific growth factors in the blood [14], the leading role among which belongs to the hepatocyte growth factor (HGF) [15, 16]. Hepatocyte growth factor is a glycoprotein that is a strong mitogen for hepatocytes and is involved in liver regeneration. It stimulates the proliferation of certain types of epitheliocytes, as well as vascular endothelial cells and melanocytes. Since its discovery, studies have identified the potential therapeutic activity of HGF for the treatment of various diseases of the liver, kidneys and lungs.

The aim was to establish the role of molecular cellular mechanisms of fibrotic processes in the liver in adolescents with juvenile idiopathic arthritis treated with MTX by determining the content of fibroblast growth factor and hepatocyte growth factor.

\section{Materials and methods}

A prospective controlled study was conducted in 68 adolescents who in 2017-2019 were treated in the Department of Cardiorheumatology of the State Institution "Institute of Child and Adolescent Health of the National Academy of Medical Sciences of Ukraine" with a diagnosis of "Juvenile Idiopathic Arthritis" (UA, ICD-X M08 according to the International Classification of Diseases 10th revision).

This paper complies with ethical standards and was approved by the Ethics Committee of the Kharkiv Medical Academy of Postgraduate Education on June 26, 2020 , protocol No. 2. This work was carried out in accordance with the Declaration of Helsinki. Informational consent was obtained from all parents.

Inclusion criteria: boys and girls aged 10 to 18 , patients with JIA (oligoarthritis and polyarthritis) treated with MTX and children whom MTX were prescribed, but they did not received it yet on the moment of this study were provided.

Exclusion criteria: children with chronic pathology of the liver, gastrointestinal tract, genetic and endocrine pathologies.

The diagnosis "Juvenile idiopathic arthritis" was established in accordance with the Order of the Ministry of Health of Ukraine dated 22.10.2012 No. 832 "On approval of a unified clinical protocol for medical care for children with juvenile arthritis". Treatment of all children was carried out in accordance with the same order.

All adolescents underwent clinical history, laboratory tests and instrumental studies to verify the diagnosis, determined the level of enzymes that are indicators of cytolytic syndrome of liver damage (AST, ALT, LDH) and liver ultrasound.

Liver fibrosis indexes calculation was performed using Microsoft Excel according to generally accepted formulas.

APRI - AST to Platelet Ratio Index:

[(AST/ULN AST) $\times 100] /$ Platelets $\left.\left(10^{9} / \mathrm{L}\right)\right]$,
FIB-4 score - (Fibrosis 4 Score):

$($ Age $\times$ AST $) /(\operatorname{Plts} \times($ sqr $($ ALT $)))$.

Blood adiponectin levels were determined by enzyme-linked immunosorbent assay using the Human Adiponectin ELISA kit manufactured by BioVendor.

Determination of BFGF and HGF levels in the blood was performed by enzyme-linked immunosorbent assay using Human bFGF ELISA and Human HGF ELISA kits made by Elabscience.

To evaluate study results, children were divided into four groups according to cumulative dose received during JIA treatment with methotrexate. Group 1 included adolescents with JIA who had just been prescribed MTX and had not yet received it ( $\mathrm{n}=18$ ), group 2 included patients who received CD MTX less than one gram $(\mathrm{n}=15)$, group 3 included adolescents, who received from 1 to 3 grams of the drug $(n=18)$, and up to 4 - adolescents who received more than 3 grams of MTX ( $n=17)$.

Statistical processing of the study materials was performed using the software package Statistica 6.0. Before statistical processing, all data were checked for normal distribution. Pearson and Spearman correlations were used. One-way ANOVA was used to statistically compare the values of the study groups. To calculate statistical significance - LSD test. Differences were considered significant at $\mathrm{p}<0.05$.

\section{Results}

68 adolescents ( 25 b oys) $(36.8 \%)$ and 43 girls $(63.2 \%)$ were examine d, with a mean age of $13.3 \pm 0.3$ years, and $23(33.8 \%)$ with oligoarthritis and $45(66.2 \%)$ with polyarthritis with complaints and objective signs relevant to JIA.

During data analysing most patients have following complaints of digestive system: nausea -28 children (42.1\%), belching $-2(3.2 \%)$, epigastric pain $-35(51.0 \%)$, pain in the pyloroduodenal area $-28(41.4 \%)$, pain in the right hypochondrium - 25 (37.7 \%), abdominal pain associated with methotrexate - in 15 (21.7\%). During abdomen palpation, pain in the right hypochondrium was in 27 (39.2 \%) patients, in the epigastrium - in 22 (31.9\%). Increased liver size obtained by palpation was detected in 50 (73.8 \%) adolescents. According to liver ultrasound in the group of patients who did not receive MTX, 30 (44\%) children showed an increase in liver size and increased echogenicity in $11(16.7 \%)$ patients. That is, according to ultrasound, most often the liver increase and its echogenicity changes were determined before taking MTX. After MTX receiving, the frequency of these manifestations decreased by half. Thus, in the group of children who received MTX up to 1 gram -15 (21.4\%) children were with liver enlargement and $5(6.7 \%)$ with increased echogenicity. In the group of children who received MTX from 1 to 3 grams, there was an increase in the liver - in $15(22.2 \%)$ and an increase in echogenicity - in 4 (5.6\%). In the group of children receiving MTX more than 3 grams - in 14 (20.4\%) children with liver enlargement, 8 (11.8\%) had an increase in echogenicity, which is almost twice more as 
much as in previous groups. This may indirectly indicate the initial signs of fibrosis.

The results of determining the content of enzymes, adiponectin, liver fibrosis indices, fibroblast growth factors and hepatocytes are given in Table 1.

Studied laboratory parameters analysis revealed a tendency to increase of BFGF level and decrease of HGF content in accordance with MTX accumulation, which may indicate a tendency of liver fibrosis and a decrease in the ability hepatocytes recovery. The level of cytolysis enzymes and adiponectin was determined within normal limits. According to liver fibrosis indexes APRI and FIB4 Score, significant changes in adolescents with the highest CD MTX (more than $3 \mathrm{~g}$ ) compared with patients who had not yet received MTX, or the cumulative dose was less than 3 grams were observed.

Correlation analysis between the studied parameters revealed the following.

When studying a cohort of children with a diagnosis of JIA who did not receive MTX, there were statistically significant correlations $(\mathrm{p}<0.05)$ of BFGF levels with ALT $(\mathrm{r}=0.512)$, FIB-4 Score index $(\mathrm{r}=0.546)$, adiponectin levels $(\mathrm{r}=0.577)$. According to this information JIA potentiates the activity of fibrotic processes in both joints and liver. This effect may not cause enough liver damage, as evidenced by the lack of correlation with HGF, i. e. the processes of liver regeneration at this stage have not yet begun.

In the group of children who received CD MTX less than 1 gram of BFGF statistically significant correlations with these indicators were not found. That is, with the start of MTX therapy there is a positive effect on liver function. Thus, the drug levels the activity of fibrotic processes in both joints and liver.

In the group of children who received MTX from 1 to 3 grams, statistically significant negative correlation of BFGF and HGF was found $(\mathrm{r}=-0.648, \mathrm{p}<0.05)$. Thus, at the same time, hepatocyte regeneration processes may begin and the liver still can compensate negative effects of JIA and MTX hepatotoxicity. That is, careful monitoring of its condition is necessary to exclude the possibility of irreversible death of hepatocytes.

In the group of children who received CD MTX more than 3 grams, there was negative statistically significant correlation of BFGF level with LDH $(r=-0.649$, $p<0.05)$. It is possible that at this stage there is a decrease in the activity of hepatocyte repair which may be associated with both the severity of JIA and the manifestation and accumulation of toxic effects of MTX.

\section{Research results discussion}

We find out that monitoring of the liver condition is extremely important during JIA treatment with methotrexate to detect early signs of liver damage. JIA can affect the liver by involving the same mechanisms as to the joints, which confirmed by positive correlations between fibroblast growth factor levels and cytolysis enzyme activity ALT, increased FIB-4 Score liver fibrosis index, and adiponectin content, which is one of the markers of inflammation and steatosis of the liver, which is consistent with previous studies [17].

Starting MTX treatment, its positive effect is observed, which is confirmed by the absence of statistically significant correlations of the studied indicators with both BFGF and HGF levels, which shows the proliferative activity of hepatocytes [14].

Reaching the CD MTX of 1 and 3 grams, there is an increase need of liver state studding, which is confirmed by the presence of a negative statistically significant correlation of BFGF and HGF. Regulatory mechanisms involvement that provoke liver regeneration when reaching 1 gram of the MTX indicates the inability of the liver to effectively perform all its functions, but it is still able to restore itself. Effective liver functioning increases the chances of further prolonged remission of the JIA, so it is extremely important to maintain its functioning. When the cumulative dose of 3 grams is reached, there is a tendency to decrease of HGF level, which may indicate a decrease in regeneration processes, which may be associated with the severity of JIA and the manifestation and accumulation of toxic effects of MTX.

Study limitations. Liver biopsy was not performed, and we cannot definitively exclude liver fibrosis and steatosis. However, morphological study can't be provided in pediatrics as a screening method which is due to its invasive nature. On the other hand, the strength of our results is that we studied liver condition in adolescents with JIA without other chronic diseases and therefore they may indicate the effect of MTX on liver function.

Table 1

Mean laboratory values in adolescents with JIA receiving methotrexate treatment

\begin{tabular}{|c|c|c|c|c|}
\hline Indicator & $1(0 \mathrm{~g} \mathrm{CD})$ & 2 (up to $1 \mathrm{~g} \mathrm{CD})$ & $3(1-3 \mathrm{~g} \mathrm{CD})$ & 4 (more than $3 \mathrm{~g}$ CD) \\
\hline BFGF $(\mathrm{pg} / \mathrm{ml})$ & $6876.4 \pm 338.1$ & $7416.1 \pm 230.1$ & $7212.1 \pm 253.7$ & $7388.4 \pm 308.6$ \\
\hline HGF $(\mathrm{pg} / \mathrm{ml})$ & $202.7 \pm 20.1$ & $163.3 \pm 11.2$ & $182.3 \pm 26.8$ & $171.5 \pm 11.5$ \\
\hline ALT (U/L) & $20.9 \pm 1.9$ & $30.1 \pm 2.8 * 1.2$ & $26.0 \pm 2.4$ & $23.6 \pm 2.03$ \\
\hline AST (U/L) & $25.1 \pm 2.3$ & $34.9 \pm 2.7 * 1.2$ & $30.5 \pm 2.8$ & $27.9 \pm 1.9$ \\
\hline LDH (U/L) & $155.7 \pm 6.4$ & $160.9 \pm 8.9$ & $146.6 \pm 8.4$ & $136.6 \pm 8.2 * 2.4$ \\
\hline APRI index & $0.296 \pm 0.03$ & $0.403 \pm 0.03 * 1.2$ & $0.354 \pm 0.4$ & $0.325 \pm 0.03 * 2.4$ \\
\hline FIB-4 Score & $0.244 \pm 0.02$ & $0.288 \pm 0.01$ & $0.281 \pm 0.02$ & $0.323 \pm 0.02 * 1.4$ \\
\hline Adiponectin (mcg/ml) & $1.53 \pm 0.1$ & $1.45 \pm 0.2$ & $1.43 \pm 0.1$ & $1.55 \pm 0.06$ \\
\hline
\end{tabular}

Note: $* 1,2,3,4-$ statistically significant difference between groups $(p<0.05)$ 
Prospects for further research. In the future, it is planned to analyse other aspects of liver dysfunction in patients with JIA. Parameters we studied can be used for timely determination of risks of irreversible pathological changes development of the liver during JIA treatment with methotrexate and timely appointment of hepatoprotective drugs.

\section{Conclusions}

1. Nausea, pain, and tenderness on palpation in the right hypochondrium were observed in $40 \%$ of adolescents with JIA treated with methotrexate. Liver enlargement was detected on palpation in $2 / 3$ of patients.

2. Ultrasound of the liver in $44 \%$ of adolescents with JIA showed its increase before treatment, and its decrease in almost half of patients on treatment.

3. The level of cytolysis enzymes (ALT, AST and LDH) in adolescents with JIA remained within normal limits, but with increasing cumulative dose of methotrex- ate there is a significant increase in liver fibrosis indices APRI index and FIB-4 Score.

4. With the accumulation of methotrexate, there was a tendency to increase the activity of fibrosis, which was confirmed by an increase in BFGF levels against the background of inhibition of liver regenerative capacity, as evidenced by a decrease in HGF.

5. Monitoring of liver fibrosis is appropriate at all stages of JIA treatment, but is most appropriate when reaching a cumulative dose of 1 and 3 grams.

Thus, timely and adequate treatment of JIA with methotrexate prevents irreversible changes in the liver, but dynamic monitoring of its functioning and early detection of signs of organ fibrosis is appropriate for timely correction of therapy.

\section{Conflict of interest}

The authors declare that they have no conflicts of interest.

\section{References}

1. Smolen, J. S., Landewé, R., Bijlsma, J., Burmester, G., Chatzidionysiou, K., Dougados, M. et. al. (2017). EULAR recommendations for the management of rheumatoid arthritis with synthetic and biological disease-modifying antirheumatic drugs: 2016 update. Annals of the Rheumatic Diseases, 76 (6), 960-977. doi: http://doi.org/10.1136/annrheumdis-2016-210715

2. Desmoulin, S. K., Hou, Z., Gangjee, A., Matherly, L. H. (2012). The human proton-coupled folate transporter. Cancer Biology \& Therapy, 13 (14), 1355-1373. doi: http://doi.org/10.4161/cbt.22020

3. Seideman, P., Beck, O., Eksborg, S., Wennberg, M. (1993). The pharmacokinetics of methotrexate and its 7-hydroxy metabolite in patients with rheumatoid arthritis. British Journal of Clinical Pharmacology, 35 (4), 409-412. doi: http://doi.org/ 10.1111/j.1365-2125.1993.tb04158.x

4. Conway, R., Carey, J. J. (2017). Risk of liver disease in methotrexate treated patients. World Journal of Hepatology, 9 (26), 1092-1100. doi: http://doi.org/10.4254/wjh.v9.i26.1092

5. Chan, E. S. L., Montesinos, M. C., Fernandez, P., Desai, A., Delano, D. L., Yee, H. et. al. (2006). Adenosine A2Areceptors play a role in the pathogenesis of hepatic cirrhosis. British Journal of Pharmacology, 148 (8), 1144-1155. doi: http://doi.org/10.1038/ sj.bjp.0706812

6. Che, J., Chan, E. S. L., Cronstein, B. N. (2007). Adenosine A2A Receptor Occupancy Stimulates Collagen Expression by Hepatic Stellate Cells via Pathways Involving Protein Kinase A, Src, and Extracellular Signal-Regulated Kinases 1/2 Signaling Cascade or p38 Mitogen-Activated Protein Kinase Signaling Pathway. Molecular Pharmacology, 72 (6), 1626-1636. doi: http://doi.org/10.1124/ mol.107.038760

7. Aithal, G. P. (2011). Hepatotoxicity related to antirheumatic drugs. Nature Reviews Rheumatology, 7 (3), 139-150. doi: http:// doi.org/10.1038/nrrheum.2010.214

8. Ortega-Alonso, A., Andrade, R. J. (2018). Chronic liver injury induced by drugs and toxins. Journal of Digestive Diseases, 19 (9), 514-521. doi: http://doi.org/10.1111/1751-2980.12612

9. Vardi, N., Parlakpinar, H., Cetin, A., Erdogan, A., Cetin Ozturk, I. (2010). Protective Effect of $\beta$-Carotene on MethotrexateInduced Oxidative Liver Damage. Toxicologic Pathology, 38 (4), 592-597. doi: http://doi.org/10.1177/0192623310367806

10. Nunes, Q. M., Li, Y., Sun, C., Kinnunen, T. K., Fernig, D. G. (2016). Fibroblast growth factors as tissue repair and regeneration therapeutics. PeerJ, 4, e1535. doi: http://doi.org/10.7717/peerj.1535

11. Maddaluno, L., Urwyler, C., Werner, S. (2017). Fibroblast growth factors: key players in regeneration and tissue repair. Development, 144 (22), 4047-4060. doi: http://doi.org/10.1242/dev.152587

12. Kang, J., Hu, J., Karra, R., Dickson, A. L., Tornini, V. A., Nachtrab, G. et. al. (2016). Modulation of tissue repair by regeneration enhancer elements. Nature, 532 (7598), 201-206. doi: http://doi.org/10.1038/nature17644

13. Kan, M., Huang, J. S., Mansson, P. E., Yasumitsu, H., Carr, B., McKeehan, W. L. (1989). Heparin-binding growth factor type 1 (acidic fibroblast growth factor): a potential biphasic autocrine and paracrine regulator of hepatocyte regeneration. Proceedings of the National Academy of Sciences, 86 (19), 7432-7436. doi: http://doi.org/10.1073/pnas.86.19.7432

14. Aw, M. M., Mitry, R. R., Hughes, R. D., Dhawan, A. (2007). Serum Hepatocyte Growth Factor and Vascular Endothelial Growth Factor in Children with Acute Liver Failure. Journal of Pediatric Gastroenterology and Nutrition, 44 (2), 224-227. doi: http:// doi.org/10.1097/mpg.0b013e31802c686b 
15. Michalopoulos, G. K. (2017). Hepatostat: Liver regeneration and normal liver tissue maintenance. Hepatology, 65 (4), 1384-1392. doi: http://doi.org/10.1002/hep.28988

16. Zhang, X., Olsavszky, V., Yin, Y., Wang, B., Engleitner, T., Öllinger, R. et. al. (2020). Angiocrine Hepatocyte Growth Factor Signaling Controls Physiological Organ and Body Size and Dynamic Hepatocyte Proliferation to Prevent Liver Damage during Regeneration. The American Journal of Pathology, 190 (2), 358-371. doi: http://doi.org/10.1016/j.ajpath.2019.10.009

17. Strashok, L. A., Pavlova, O. S. (2020). Determination of liver damage indices APRI and FIB-4 score in adolescents with juvenile idiopathic arthritis. Child`s health, 15 (3), 154-159. doi: http://doi.org/10.22141/2224-0551.15.3.2020.204549

Received date 07.08.2020

Accepted date 10.09.2020

Published date 30.09.2020

Olga Pavlova, PhD Student, Department of Adolescent Medicine, Kharkiv Medical Academy of Postgraduate Education, Amosova str., 58, Kharkiv, Ukraine, 61176

E-mail: ospavlova@mail.ua

UDC 616.697:616.69:616.43-008.6

DOI: $10.15587 / 2519-4798.2020 .212118$

\section{STUDY OF THE EFFECT OF THE XENOBIOTIC HORMONE ON THE REPRODUCTIVE FUNCTION OF RATS}

\section{N. Kononenko, A. Minukhin, V. Chikitkina}

The aim is to study the effect of the xenobiotic of Nonylphenol on functional and morphological indices of the reproductive system of males of rats.

Materials and methods. Experimental modelling of gonadotoxic effects of the xenobiotic was performed by inhalation of Nonylphenol at a concentration of $500 \mathrm{mg} / \mathrm{m}^{3}$ for 30 days, 5 days a week, 5 hours a day in conditions of the seed chamber. Male rats ejaculate were obtained by the method of electrostimulation seed tubercle in the mucous membrane of the rectum. The number of sperm in the ejaculate and their mobility were determined in the Hemocytometer 1, 7, 14 and 30 days after the start of the experiment. Macroscopic study of the testes included the assessment of external signs of damage, the determination of the mass and length of the testes.

Results. It is established that the introduction of Nonylphenol to rats males leads to a gradual dynamical reduction of the total number and the number of mobile forms of sperm cells compared to intact animals. At the end of the experiment the number of mobile forms of sperm was 2.1 times below the original data. Long-term effect of Nonylphenol has also led to a significant decrease of body weight growth, which confirms its high toxicity. In the study found that intoxication in Mature rats Nonylphenol were significant changes in the reproductive system, which is primarily characterized by decreased weight of the testes and the appendages of the testis, and changes in linear dimensions of the bodies downward. These violations indicate a feminizing effect of this hormone-like xenobiotic and its role in the development of male infertility.

Conclusions. Quantitative and qualitative parameters of the ejaculate of sexually mature male rats can serve as a valid criterion for adaptive and maladaptive processes occurring in the organism under the influence of the xenobiotic. In conditions of prolonged exposure to Nonylphenol (within 30 days) the changes in mass of the testes and reduce their linear dimensions

Keywords: Nonylphenol, reproductive system, gonads, spermatogenesis

Copyright (C) 2020, N. Kononenko, A. Minukhin, V. Chikitkina.

This is an open access article under the CC BY license (http://creativecommons.org/licenses/by/4.0).

\section{Introduction}

Millions of years living organisms seen only with the «familiar cues» of the environment, as the basis on which in evolution formed their functional relationships with the outside world, and between organs and cells of the organisms themselves were the chemical and physical factors that are strictly conform to the composition and level of specific terrestrial environment: chemical com- position of seawater, where life began, atmospheric gases, temperature fluctuations, light, atmospheric pressure, levels of natural radiation, etc. At the same time, on its basis, mechanisms of neutralization and excretion of a substance foreign to it were formed. All this ensured the effective functioning and reproduction of organisms and their adaptation to the changing conditions of existence due to fluctuations in climate and other factors, the 